\title{
Differences in Photoreceptor Processing Speed for Chromatic and Achromatic Vision in the Bumblebee, Bombus terrestris
}

\author{
Peter Skorupski and Lars Chittka \\ Queen Mary University of London, Research Centre for Psychology, School of Biological and Chemical Sciences, Mile End Road, London E1 4NS, United \\ Kingdom
}

\begin{abstract}
Fast detection of visual change can be mediated by visual processes that ignore chromatic aspects of the visual signal, relying on inputs from a single photoreceptor class (or pooled input from similar classes). There is an established link between photoreceptor processing speed (in achromatic vision) and visual ecology. Highly maneuverable flies, for example, have the fastest know photoreceptors, relying on metabolically expensive membrane conductances to boost performance. Less active species forgo this investment and their photoreceptors are correspondingly slower. However, within a species, additional classes of photoreceptors are required to extract chromatic information, and the question therefore arises as to whether there might be within-species differences in processing speed between photoreceptors involved in chromatic processing compared with those feeding into fast achromatic visual systems. We used intracellular recording to compare light-adapted impulse responses in three spectral classes of photoreceptor in the bumblebee. Green-sensitive photoreceptors, which are known to provide achromatic contrast for motion detection, generated the fastest impulse responses (halfwidth, $\Delta t=7.9 \pm 1.1 \mathrm{~ms}$ ). Blue- and UV-sensitive photoreceptors (which are involved in color vision) were significantly slower (9.8 \pm 1.2 and $12.3 \pm 1.8 \mathrm{~ms}$, respectively). The faster responses of green photoreceptors are in keeping with their role in fast achromatic vision. However, blue and UV photoreceptors are still relatively fast in comparison with many other insect species, as well as vertebrate cones, suggesting a significant investment in photoreceptor processing for color vision in bees. We discuss this finding in relation to bees' requirement for accurate learning of flower color, especially in conditions of variable luminance contrast.
\end{abstract}

\section{Introduction}

The purpose of vision is to accurately perceive and rapidly apprehend change in the world (Jacobs, 2003). However, this can be metabolically expensive. Detailed spatial perception requires an array of many photoreceptors, whereas rapid appreciation of change in visual detail requires that these photoreceptors respond quickly. Photoreceptors, however, are expensive cells, fast ones even more so (Niven et al., 2007). This is because fast changes in membrane voltage need large membrane conductances, which means more active transport is needed to maintain the concentration gradients sustaining such signaling. If metabolic cost is a major constraint on brain design (Laughlin et al., 1998), then a visual system should only invest in such expensive conductances where temporal accuracy is at a premium.

Photoreceptor temporal resolution is clearly a limiting factor in motion vision (Land, 1999) and highly maneuverable insects invest in faster photoreceptors than those of slower flying species (Laughlin and Weckstrom, 1993). However, there would seem to

\footnotetext{
Received Nov. 15, 2009; revised Dec. 25, 2009; accepted Jan. 29, 2010.

We thank Simon Laughlin and Jeremy Niven for stimulating discussion. We also thank Syngenta Bioline for providing the bees used in our experiments.

Correspondence should be addressed to Peter Skorupski at Queen Mary University of London, Research Centre for Psychology, School of Biological and Chemical Sciences, Mile End Road, London E1 4NS, UK. E-mail: p.skorupski@ qmul.ac.uk.

DOI:10.1523/JNEUROSC1.5700-09.2010

Copyright $\odot 2010$ the authors $\quad 0270-6474 / 10 / 303896-08 \$ 15.00 / 0$
}

be less demand for high temporal resolution in color vision, and the temporal resolution of color vision is lower than that of achromatic vision in both humans (Boynton, 1979) and honeybees (Srinivasan and Lehrer, 1985).

In mammals, a dense array of fairly broadband receptors (which are also used in high acuity spatial vision) samples the middle to long wavelength end of the visible spectrum, whereas a much sparser receptor array samples the short wave end, thereby adding a chromaticity signal. This is thought to represent the ancestral mammalian color vision system (Regan et al., 2001). Insect ommatidia are also dominated by long-wave receptors; six of nine receptor cells per ommatidium are "green" receptors in many species, including the Hymenoptera (Wakakuwa et al., 2007). In bees, green-sensitive photoreceptors drive many achromatic visual functions, whereas color vision depends on comparison of signals in green, blue, and UV receptors (Lehrer et al., 1988; Briscoe and Chittka, 2001). Thus, long-wave receptors drive achromatic visual systems, whereas less numerous, shorter wave-sensitive receptors add color to the visual signal. Computation of color requires comparison of signals from these different spectral classes of photoreceptor, which implies the need for some matching of photoreceptor frequency responses. However, since all photoreceptor signals could be low-pass filtered at the comparison stage (Anderson and Laughlin, 2000), photoreceptor spectral classes feeding into chromatic systems could potentially economize on signaling cost by having slower response times 
than photoreceptors feeding into fast achromatic systems. This assumes that separate downstream systems process color and motion (and that these systems can have different time constants), an assumption for which there is psychophysical and behavioral evidence in both mammals and insects (Srinivasan and Lehrer, 1985; Livingstone and Hubel, 1987; Srinivasan and Lehrer, 1988; Spaethe et al., 2001).

We have compared the response times of green-, blue-, and UV-sensitive photoreceptors in the bumblebee Bombus terrestris. All spectral classes respond faster with light adaptation, but the highest response speeds are attained by green photoreceptors, followed by blue, and then UV, both of which are significantly slower than green photoreceptors, even when fully light-adapted.

\section{Materials and Methods}

Preparation. Recordings were made from bumblebee workers (Bombus terrestris dalmatinus) from Syngenta Bioline. Bees were cold-anesthetized in the freezer compartment of a refrigerator for 10-14 min and prepared for recording as previously described (Skorupski et al., 2007). Recording electrodes were pulled from $1 \mathrm{~mm}$ diameter borosilicate glass capillaries with a Flaming/Brown P-97 (Sutter Instruments) and filled with $2 \mathrm{M} \mathrm{KCl}$. Resistances were $100-150 \mathrm{M} \Omega$ when inserted into the retina.

Stimulation and recording. Light stimuli were delivered by focusing the source onto the opening of a liquid light guide, the other end of which was mounted in a Cardan arm device $\sim 6 \mathrm{~cm}$ from the eye. For initial identification of cells, the source was a $300 \mathrm{~W}$ tungsten-halogen lamp. A monochromator (M300; Bentham) and shutter (Uniblitz LS2; Vincent Associates) generated $10 \mathrm{~ms}$ flashes of monochromatic light with peak wavelengths $\sim 360,440$, and $540 \mathrm{~nm}$. Responses to such flashes quickly and unambiguously distinguish green, blue, and UV photoreceptors in the bumblebee (Skorupski et al., 2007). During most experiments the light source was a high-intensity LED, with a peak wavelength in the near-UV (360 nm, $15 \mathrm{~nm}$ half-width FoxUV; DComponents) or blue (470 nm, $22 \mathrm{~nm}$ half-width) region of the spectrum.

Following impalement and initial identification, cells were usually left in the dark for 5-10 min before recording commenced. During experiments, cells were exposed to adapting illuminations for periods ranging from 150 to $300 \mathrm{~s}$ interspersed with somewhat longer durations to allow readaptation to dark conditions and recovery of membrane potential. Voltage fluctuations were conspicuous in recordings of photoreceptor membrane potential in the dark (photoreceptor noise, presumably reflecting summed quantum events; see Results). However, in all but a few cases (which always involved cells that were held for $>2 \mathrm{~h}$ ) the pattern of noise fluctuation was too complex to allow reliable visual identification of discrete events (quantum bumps). Although cells were not studied further unless the mean membrane potential recovered to within $5 \mathrm{mV}$ of its starting level, it nevertheless seems likely that full, deep-dark adaptation would require considerable periods of time, which would be incompatible with the $60 \mathrm{~min}$ or so of recording time typical for most cells in this study. Consequently, recordings from cells in this study in the absence of adapting illumination are best regarded as "near-dark adapted" rather than "deeply dark adapted" (Laughlin and Weckstrom, 1993). For this reason we did not attempt to calibrate the light stimulus in terms of receptor-specific quantum catch (effective photons per receptor per second) (Howard et al., 1987). Instead, we measured the radiant intensity of the output light source inside the recording set-up with a spectrophotometer (Avaspec 2048; Avantes), and we express stimulating intensities in relative log units.

The peak spectral sensitivities of blue and green receptors are $\sim 436$ and $532 \mathrm{~nm}$, respectively; at $470 \mathrm{~nm}$ the relative spectral sensitivities are $\sim 0.39$ for blue and 0.53 for green (Skorupski et al., 2007). UV receptors have negligible sensitivity at this wavelength, or in some cases even generate negative responses (Skorupski et al., 2007). Saturating responses in both blue and green photoreceptors were obtained with a flash intensity corresponding to $\sim 2 \times 10^{14}$ photon $\mathrm{cm}^{-2} \mathrm{~s}^{-1}$ from the $470 \mathrm{~nm}$ LED. All stimulating intensities are expressed in relative log units normalized to this intensity. Cells accepted for analysis generated peak responses

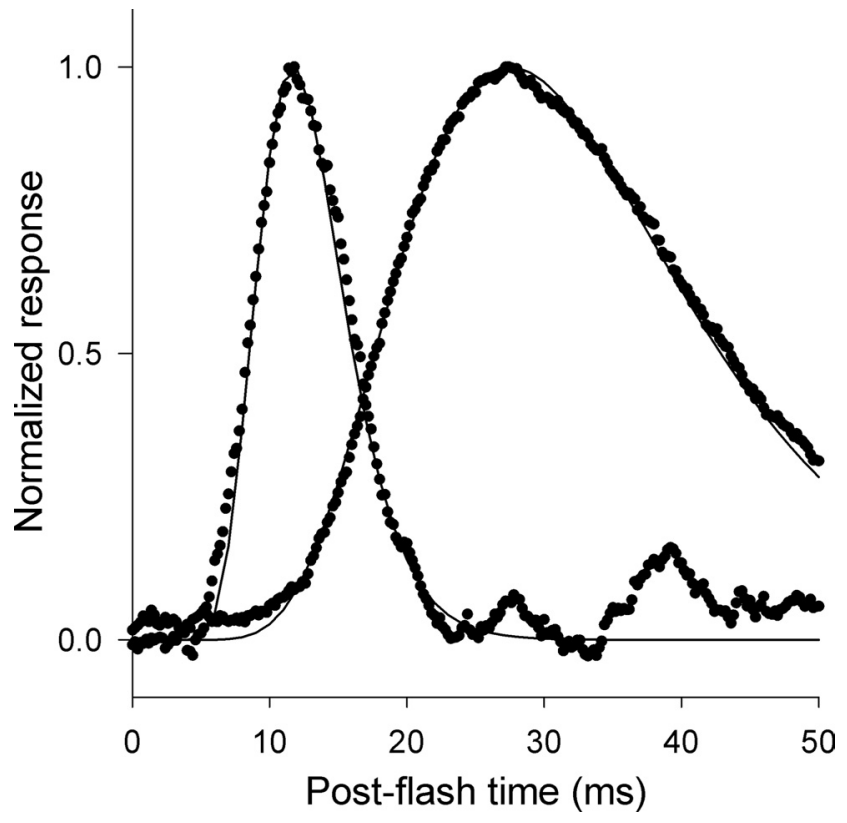

Figure 1. Photoreceptor impulse responses can be fitted with the lognormal function. Normalized bumblebee green photoreceptor impulse responses to $0.1 \mathrm{~ms}$ flashes of light in lightadapted (left-hand peak) and near-dark-adapted (right-hand peak) states. Lines are fits of the lognormal function to the data points. Adapting illumination was $-1.9 \log$ units at $360 \mathrm{~nm}$, resulting in a mean steady-state depolarization from rest of $18.8 \mathrm{mV}$. For this cell, the time to peak, $t_{p}$, decreased from $27.5 \mathrm{~ms}$ in the dark to $11.8 \mathrm{~ms}$ under light adaptation, whereas corresponding values for the skewness parameter, $\sigma$, were 0.38 and 0.27 .

$\geq 50 \mathrm{mV}$ for blue and green photoreceptors. The spectral sensitivity of the UV receptor is $\sim 340 \mathrm{~nm}$ (Skorupski et al., 2007) and at $360 \mathrm{~nm}$ its sensitivity is considerably higher than that of either blue or green photoreceptors. The maximum light intensity from the $360 \mathrm{~nm}$ LED was -1.7 $\log$ units, which generated peak flash responses of $\geq 40 \mathrm{mV}$ from UV photoreceptors.

The LEDs were driven by software-generated voltage sequences using a micro1401 lab interface (CED) and a voltage-to-current converter. Flash intensity and adapting light intensity were controlled by varying the LED voltage (using calibration curves measured with the spectrophotometer). Neutral density filters were also used for large reductions in light intensity. Photoreceptor responses were filtered at $1 \mathrm{kHz}$, digitized at $5 \mathrm{kHz}$, and written to disk.

Analysis. We assessed photoreceptor response times in two ways. First, we measured the photoreceptor impulse response by averaging responses to 100-400 brief, low-intensity flashes. Stimulus amplitude was adjusted by varying flash duration $(0.1-3.0 \mathrm{~ms})$ and voltage, so that peak response amplitude was $\leq 1.5 \mathrm{mV}$ (typically $0.4-0.8 \mathrm{mV}$ ). By keeping response amplitude low, linearity can be assumed and the response can be well fitted with a lognormal function (Howard et al., 1984) as follows:

$$
v(t)=\exp \left(-\left(\ln \left(t / t_{\mathrm{p}}\right)\right)^{2} / 2 \sigma^{2}\right) .
$$

In this equation, $t_{\mathrm{p}}$ is the time to peak and $\sigma$ is a shape parameter. Smaller $\sigma$ values produce more symmetric responses and larger values more positively skewed responses. For a given $t_{\mathrm{p}}$, therefore, increasing $\sigma$ increases response duration. Response durations may usefully be compared by means of the half-width, $\Delta t$, which, to an accuracy of $1 \%$ (Howard et al., 1984), is given by the following:

$$
\Delta t=2.35 t_{\mathrm{p}} \sigma .
$$

Impulse responses were obtained in the dark and in light-adapted conditions at 1-4 different adapting intensities. Intensity of adapting illumination (which ranged from -5.2 to $-1.9 \log$ units at $360 \mathrm{~nm}$ and -4.4 to $-1.2 \log$ units at $470 \mathrm{~nm}$ ) was controlled by maintained LED voltages 
Table 1. Fitted parameters for bumblebee photoreceptor impulse responses in the dark- and light-adapted states

\begin{tabular}{|c|c|c|c|c|c|c|c|c|}
\hline \multirow[b]{2}{*}{ Spectral type } & \multicolumn{4}{|l|}{ Dark adapted } & \multicolumn{4}{|l|}{ Light adapted } \\
\hline & $t_{\mathrm{p}}(\mathrm{ms})$ & $\sigma$ & $\Delta t(\mathrm{~ms})$ & $n$ & $t_{\mathrm{p}}(\mathrm{ms})$ & $\sigma$ & $\Delta t(\mathrm{~ms})$ & $n$ \\
\hline G & $32.2 \pm 4.77$ & $0.36 \pm 0.017$ & $27.32 \pm 4.15$ & 11 & $12.53 \pm 1.59$ & $0.27 \pm 0.019$ & $7.88 \pm 1.09$ & 18 \\
\hline B & $30.93 \pm 4.55$ & $0.33 \pm 0.028$ & $24.23 \pm 5.27$ & 11 & $13.94 \pm 1.02$ & $0.30 \pm 0.021$ & $9.76 \pm 1.20$ & 19 \\
\hline UV & $43.7 \pm 9.24$ & $0.31 \pm 0.03$ & $32.13 \pm 6.68$ & 5 & $15.67 \pm 1.36$ & $0.33 \pm 0.023$ & $12.33 \pm 1.77$ & 7 \\
\hline
\end{tabular}

Values are mean \pm SD. G, Green; B, blue.

and neutral density filters, and impulse responses at each adaptation level were generated by brief, pulsed increments of the LED voltage.

Second, we computed power spectra from photoreceptor noise recorded over a similar range of adapting light intensities and analyzed the frequency bandwidth of the noise power. Continuous sequences of photoreceptor noise were Fourier-transformed in blocks of 2048 points (after subtraction of membrane potential) using the fast Fourier transform algorithm in Spike2. Given the sampling rate of $5 \mathrm{kHz}$, this results in block lengths of $0.4096 \mathrm{~s}$ and a frequency resolution of $2.44 \mathrm{~Hz}$. Power spectra are averages of $60-150$ such blocks from 25 to $60 \mathrm{~s}$ of continuous photoreceptor noise recording.

An estimate of the unitary event duration, $\tau$, underlying photoreceptor noise can be obtained by fitting the power spectrum with a Lorentzian (Howard et al., 1984; Vorobyev et al., 2001) as follows:

$$
P(f)=A /\left(1+(2 \pi f \tau)^{2}\right) .
$$

The corner frequency $\left(f_{\mathrm{c}}\right)$ can then be estimated as the frequency at which the power drops by half (i.e., $P\left(f_{\mathrm{c}}\right)=A / 2$ ).

\section{Results}

\section{Impulse responses}

Impulse responses from green, blue, and UV photoreceptors were generally well fitted with the lognormal function (Fig. 1). In the absence of light adaptation, there were no significant differences in response duration between photoreceptor classes. However, following light adaptation significant differences emerged: response duration decreased in all three classes, but the fastest responses were recorded in green photoreceptors.

In the near-dark-adapted condition, there was considerable scatter in the data from cell to cell, with half-widths $(\Delta t)$ ranging from $\sim 19$ to $41 \mathrm{~ms}$. There was a trend for longer impulse response durations in UV receptors $(\Delta t=32.1 \pm 6.7 \mathrm{~ms})$ compared with green $(\Delta t=27.3 \pm 4.2 \mathrm{~ms})$ or blue $(\Delta t=24.2 \pm 5.3$ $\mathrm{ms}$ ) but this was not significant. Similarly, there were no differences in the estimated values for the time to peak, $t_{\mathrm{p}}$, and the shape parameter, $\sigma$ (Table 1 ).

During adaption to light of either 360 or $470 \mathrm{~nm}$, cells from all three receptor classes reached maximum steady-state depolarizations of $30 \mathrm{mV}$ or more (Fig. 2), although the intensity required varied, as expected, with spectral sensitivity. We also noted that cells were more likely to be lost during intense light adaptation; consequently, in most experiments the highest intensities used were restricted to those that elicited steady-state depolarizations of $20-25 \mathrm{mV}$.

The decrease in impulse response duration in all three photoreceptor classes following light adaptation was associated with a decrease in both the time to peak $\left(t_{\mathrm{p}}\right)$ and the skewness parameter $(\sigma)$, resulting in faster, more symmetrical impulse responses in light-adapted cells. This effect tended to saturate at adapting intensities sufficient to depolarize a cell $15-20 \mathrm{mV}$ from rest (Fig. 2). Moreover, at these levels of light adaptation there also emerged significant differences between the three spectral classes of receptor. Comparing all impulse responses where the lightadapted resting membrane potential was $>15 \mathrm{mV}$ depolarized from the dark resting value, the fastest (shortest duration) im-

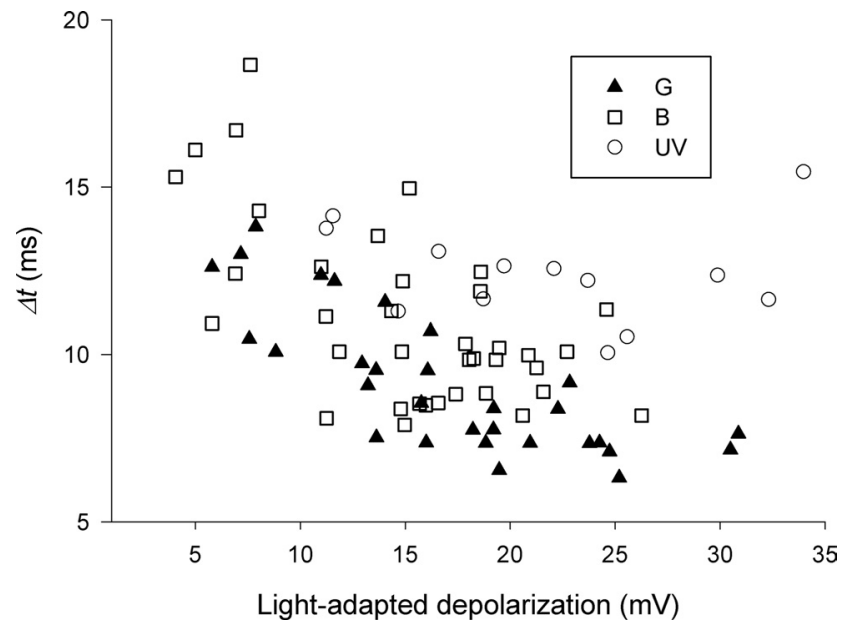

Figure 2. Impulse response half-width plotted against light-adapted membrane potential for green $(\mathbf{\Delta})$, blue $(\square)$, and UV $(\bigcirc)$ photoreceptors. Each data point represents a single averaged impulse response.

Table 2. Significance of variation of lognormal parameters among spectral classes of photoreceptors

\begin{tabular}{llll}
\hline Test & $t_{\mathrm{p}}$ & $\sigma$ & $\Delta t$ \\
\hline G-UV & 0.00029 & 0.000060 & 0.00028 \\
G-B & 0.0067 & 0.000083 & 0.000035 \\
B-UV & 0.014 & 0.0039 & 0.0062
\end{tabular}

$\overline{p \text { values for unpaired } t \text { test. For all values except } \mathrm{B}-\mathrm{UV} \text {, the difference in } t_{\mathrm{p}} \text { remains significant after Bonferroni }}$ correction for three-way comparison. $G$, Green; B, blue.

pulse responses were generated in green receptors $(\Delta t=7.88 \pm$ $1.09 \mathrm{~ms})$, followed by blue $(\Delta t=9.76 \pm 1.20 \mathrm{~ms})$, with the slowest occurring in UV receptors $(\Delta t=12.33 \pm 1.77 \mathrm{~ms})$. Data for all fitted parameter values are presented in Table 1. All three parameters differed significantly between photoreceptor classes in the light-adapted state (Table 2).

\section{Photoreceptor noise}

Prominent noise fluctuations were evident in photoreceptor responses to low-intensity flashes and also in the resting membrane potential in the dark (Fig. 3A). The amplitude of this noise (expressed as SD of membrane voltage) increased with low-intensity light adaptation (intensities sufficient to generate a steady-state photoreceptor depolarization of $\leq 10 \mathrm{mV}$ ), but decreased again with progressively higher adapting intensities (Figs. $3 \mathrm{~B}, \mathrm{C}$ ). This is evident in the noise power spectra for all three photoreceptor classes (Fig. 4), where the power in the low-frequency range $(<20$ $\mathrm{Hz}$ ) decreases with increasing adapting light intensity in all photoreceptors. This reflects the membrane potential becoming less "bumpy" as the light response is generated by the summation of many more discrete events, so that individual events are less discernible as more are summated (Dodge et al., 1968). At the same time, the power spectra show an increase in the proportion of the noise power found in higher frequencies. This is reflected both in 
A

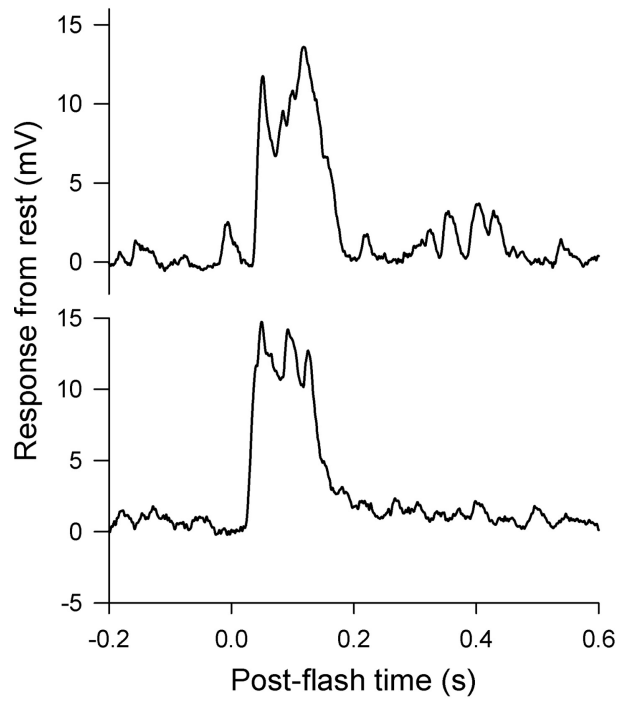

B

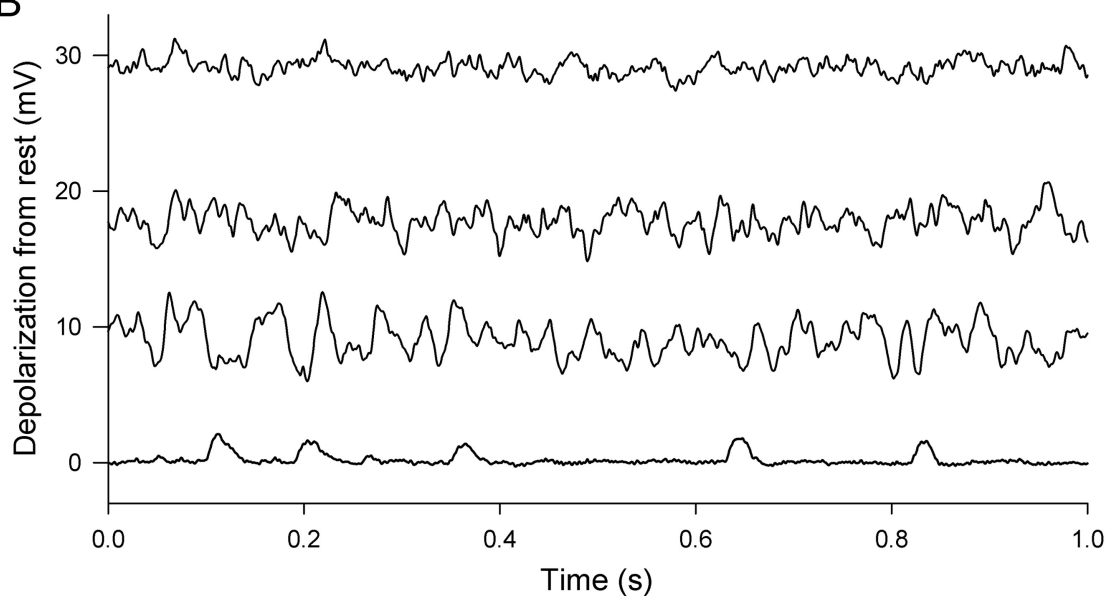

Figure 3. A, Photoreceptor dark noise and responses to dim flashes for a UV receptor (upper trace) and a green receptor (lower trace). Ten millisecond flashes, $360 \mathrm{~nm},-4.3$ and $-3.9 \mathrm{log}$ units respectively. $\boldsymbol{B}$, Photoreceptor noise at increasing levels of steady adapting illumination. C, Photoreceptor noise in UV receptors (measured as SD of membrane potential) plotted for dark and three levels of increasing adapting illumination (LA1-LA3).

a decrease in the rate of high-frequency "roll-off" and also in an increase in the bandwidth of the region where the frequency response is relatively flat. Figures $4 A-C$ display averaged noise spectra recorded from green, blue, and UV photoreceptors (three to eight cells from each class) at three levels of adapting illumination of increasing intensity (see Fig. 4 legend for details).

Noise power spectra also differed between the three photoreceptor classes. The proportion of high-frequency noise power was greatest in green and smallest in UV photoreceptors (Fig. $4 D$ ). For purposes of comparison, spectra from individual cells at a given level of light adaption were fitted with the Lorentzian (Eq. 3) to estimate event duration $(\tau)$. As would be expected, $\tau$ decreased with light adaptation in all photoreceptors, although this effect, like impulse response duration, tended to saturate with adapting light intensities sufficient to induce a steady-state depolarization in the region of $15-20 \mathrm{mV}$ depolarized from the dark resting value.

In Figure 5, for purposes of illustration, estimates of $\tau$ from individual power spectra were used to calculate the corner frequency $f_{\mathrm{c}}$ (the frequency at which the noise power drops to half its maximum). The corner frequency is plotted against light- adapted membrane potential for three (UV cells) or two (blue and green cells) intensities of adapting light at $360 \mathrm{~nm}$ (Fig. 5A). A similar plot, over four intensities of adapting light at $470 \mathrm{~nm}$, is shown for blue and green cells in Figure $5 \mathrm{~B}$. At low adapting intensities there is little difference between photoreceptors, but at moderate to high levels a pattern emerges where green photoreceptors show the highest frequencies, followed by blue, and then UV with the lowest frequencies. Mean values for the estimated event duration from all noise spectra where the light-adapted membrane potential was $>15 \mathrm{mV}$ depolarized from rest showed a clear order: $\tau=$ $2.6 \pm 0.3 \mathrm{~ms}$ (green); $\tau=3.4 \pm 0.6 \mathrm{~ms}$ (blue); $\tau=5.1 \pm 0.5 \mathrm{~ms}$ (UV).

\section{Responses to light offset}

Fast vision requires good temporal resolution of contrast changes in either the positive or negative direction. During these experiments we noticed large-scale differences to illumination step changes, including long-lasting after-hyperpolarizations and depolarizations that were most prominent in green photoreceptors. We also observed that the initial recovery of membrane potential following adapting light offset was significantly steeper in green compared with UV photoreceptors. In 12 cells (green $=5$; blue $=4$; UV $=3$ ) from two animals, we compared responses to large negative contrast steps generated by switching off the adapting light for 30 ms (assuming a photoreceptor acceptance angle of $1^{\circ}$, this would correspond to an angular velocity of $\left.\sim 33^{\circ} \mathrm{s}^{-1}\right)$. Responses were recorded following at least $60 \mathrm{~s}$ light adaptation, and the adapting intensity was adjusted so that the light adapted membrane potential was $20 \pm 3 \mathrm{mV}$. Figure 6 shows averaged responses to these steps normalized to the mean light-adapted membrane potential. Both the amplitude and time course of these "off" responses varied between photoreceptor classes. UV photoreceptor responses were smallest and slowest, repolarizing to within $\sim 50 \%$ of the dark resting potential within $40 \mathrm{~ms}$ of light off. Blue photoreceptors repolarized to within $20 \%$ of the dark resting level. In contrast, green photoreceptors hyperpolarized fully to the dark resting level within $25 \mathrm{~ms}$, and subsequently generated a hyperpolarizing undershoot of 4-8 $\mathrm{mV}$ (Fig. 6).

\section{Discussion}

Spatial resolution of color vision is lower than achromatic vision in both humans (Snowden et al., 2006) and bees (Giurfa and Lehrer, 2001; Skorupski et al., 2006). Temporal resolution is also lower, as inferred from critical flicker-fusion frequencies (Boynton, 1979; Srinivasan and Lehrer, 1985; Jiang et al., 2007). Here we demonstrate that bumblebee green photoreceptors, which drive fast achromatic vision, exhibit better temporal resolution 
than blue or UV photoreceptors, thought to be involved predominantly in chromatic processing.

In the Diptera, fast-flying species such as blowflies improve photoreceptor frequency response by means of slowly activating delayed rectifier potassium conductances, which are not found in photoreceptors from slower species, such as crane flies (Laughlin and Weckstrom, 1993). Within the same retina, photoreceptors of different classes may also show differences in voltage-dependent potassium conductance (Anderson and Hardie, 1996), suggesting a role for such conductances in tuning photoreceptor performance both between and within species. In the bumblebee, we have not yet established the electrophysiological basis for differences in photoreceptor response times; however, an undershoot in membrane potential on release from depolarization [observed in response to brief pulses of darkness in green, but not blue or UV photoreceptors (Fig. 6)] is strongly suggestive of voltage-dependent potassium conductance.

\section{Comparison with other species}

By comparison with other insect species from several orders (Table 3), bumblebee photoreceptors are relatively fast, regardless of spectral class. Only fast-flying flies outperform bumblebee green and blue photoreceptors, and even UV receptors from bumblebees compare favorably with dragonfly, hoverfly, and mantid photoreceptors, and easily outperform those from locust and cricket. In the study of Howard et al. (1984), photoreceptors were not identified according to spectral class, but it is likely that most recordings were from middle to long wave-sensitive, relatively broadband receptors, equivalent to dipteran R1-R6 or hymenopteran green photoreceptors. The only previous study, to our knowledge, to have explicitly compared photoreceptor frequency response within the same retina is that of Anderson and Laughlin (2000). This study found that blowfly photoreceptors thought to be involved in chromatic signaling (R7-R8 photoreceptors, which show a complex pattern of relatively narrowly tuned spectral sensitivities) were less efficient at tracking high-frequency changes in visual contrast than achromatic photoreceptors (R1-R6 photoreceptors, which are double-peaked, broadband photoreceptors feeding into fast achromatic systems). However, the precise role of photoreceptor spectral sensitivities in fly color vision has yet to be established.

Vertebrate rods are much slower than insect photoreceptors, with response durations (integration times) to brief dim flashes of $300 \mathrm{~ms}$ or more (Baylor, 1987). Responses of dark-adapted turtle cones to such flashes peak within 100-150 ms, decreasing to $\sim 50 \mathrm{~ms}$ with light adaptation (Baylor and Hodgkin, 1974). Macaque cone responses to similar stimuli peak in $40-50 \mathrm{~ms}$, with no apparent change on light adaptation (Baylor et al., 1987; Schneeweis and Schnapf, 1999), or with a decrease from dark
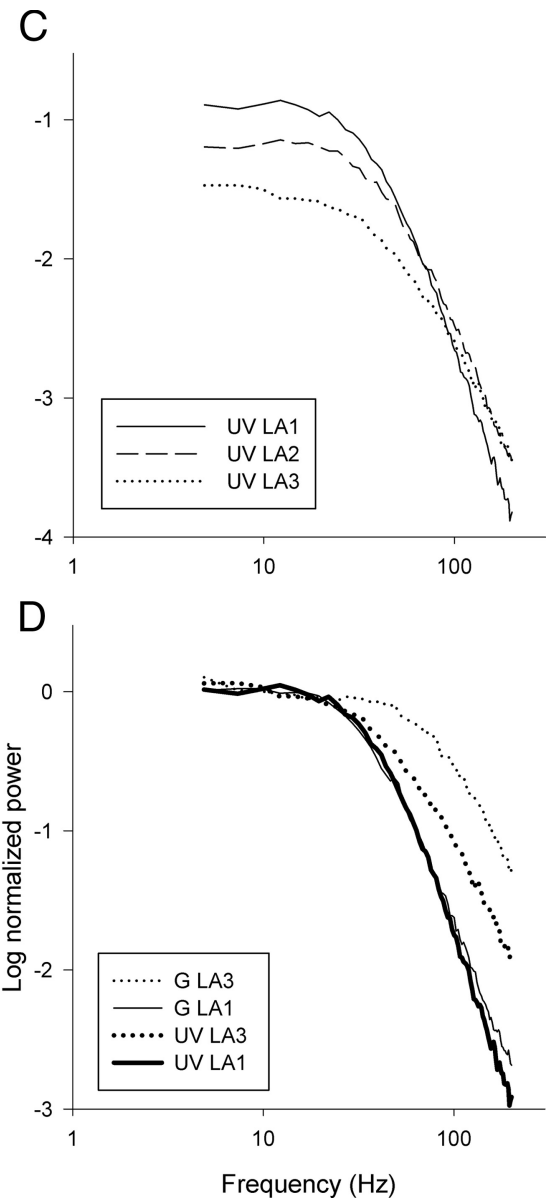

Frequency $(\mathrm{Hz})$

Figure 4. Averaged noise power spectra recorded from green (G), blue (B), and UV cells. $A$, Green cells at $-4.4 \log$ units (470 $\mathrm{nm} ; n=5$ cells; solid line, LA1), -3.2 ( $n=6$; dashed line, LA2), and $-1.2(n=8$; dotted line, LA3). $B$, Spectra for blue cells at $-2.2 \log$ units ( $n=4$ cells in each case). D, Power spectra for green (thin lines) and UV (thick lines) photoreceptors, normalized to mean of frequency bins $<20 \mathrm{~Hz}$ and overlaid for comparison. LA1-LA3, Levels of increasing adapting illumination.

response duration of $\sim 50$ to $\sim 40 \mathrm{~ms}$ with intense light adaptation (Dunn et al., 2007). Interestingly, ground squirrels seem to have the fastest cones yet recorded, with impulse response peak times of $\sim 20 \mathrm{~ms}$ (Kraft, 1988).

Table 3 suggests that bumblebees are investing relatively heavily in maintaining the frequency response of their color vision (albeit at a lower level than for achromatic vision), since, presumably, the slower photoreceptors of mantids and locusts are more economical in terms of membrane conductance. What ecological factors might dictate bees' needs for fast achromatic (and relatively fast chromatic) vision?

Worker bees do not engage in fast visual pursuit of moving targets, but they do fly rapidly over considerable distances, and learn nest and food source locations in a visually complex landscape. This is accomplished by systematic scanning of the landscape in "orientation flights," which can result in estimated retinal image velocities of $100-200^{\circ}{ }^{-1}$ (Zeil et al., 1996). The retinal image is significantly compromised by motion blur when image angular velocity exceeds the ratio of photoreceptor acceptance angle to photoreceptor integration time, and at velocities twice this value photoreceptor contrast modulation is effectively reduced to zero (Land, 1999). Assuming $1^{\circ}$ for acceptance angle, the motion blur would become significant at image angular velocities greater than $\sim 127^{\circ} \mathrm{s}{ }^{-1}$ in green photoreceptors, $100^{\circ} \mathrm{s}{ }^{-1}$ in blue, and $80^{\circ} \mathrm{s}^{-1}$ in UV. Green receptors, therefore, would still 

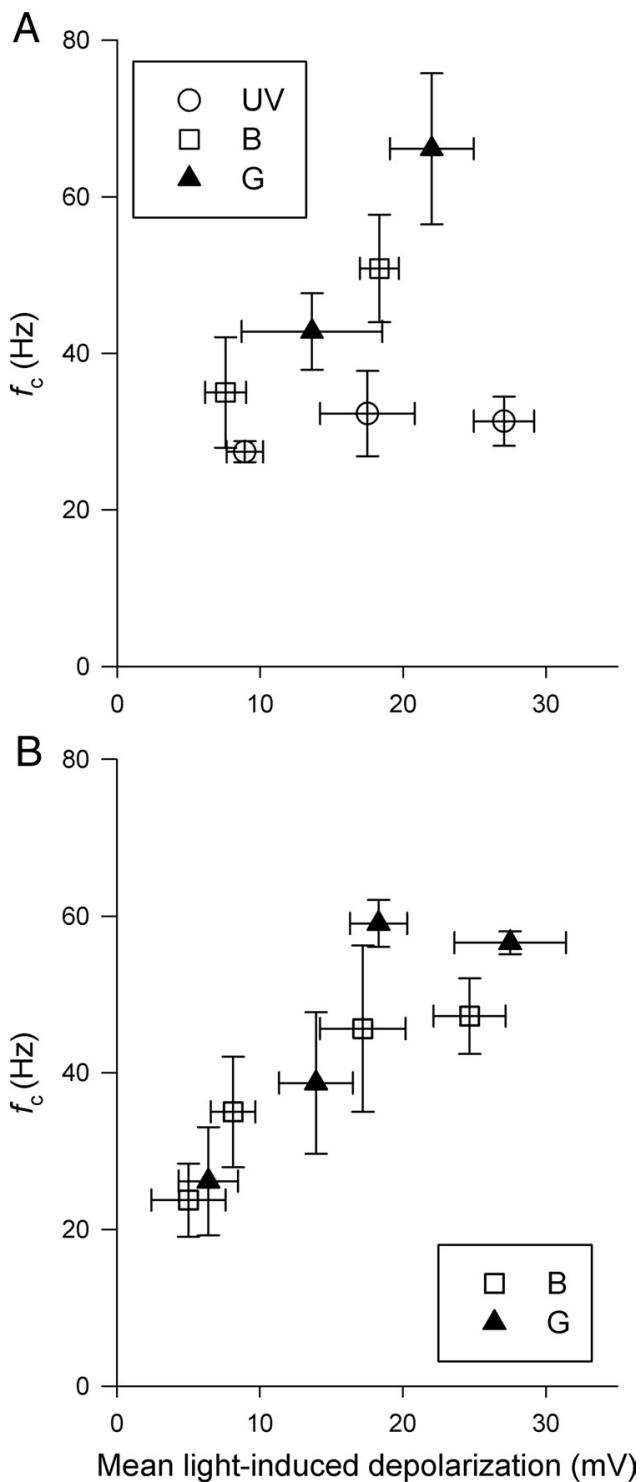

Figure 5. Estimated corner frequency of photoreceptor noise power plotted against mean light-adapted membrane potential for green $(\mathbf{\Delta})$, blue $(\square)$, and UV $(\bigcirc)$ photoreceptors. A, Adapting light $(360 \mathrm{~nm})$; data points correspond (from left to right) to intensities in relative log units of $-4,-3.3$, and -2.2 (UV) and -3.1 and -1.9 (blue and green). $\boldsymbol{B}$, Adapting light $(470 \mathrm{~nm})$; relative intensities of $-4.4,-3.2,-2.4$, and -1.2 (blue and green).

be able to provide a useful contrast signal over the full range of angular velocities experienced during orientation flights. Because bees use achromatic (green photoreceptor) contrast in motion and form perception, and because bees can also perceive depth and form through motion cues (Lehrer et al., 1988; Giurfa and Lehrer, 2001; Lehrer and Campan, 2005), the benefit of a fast response time for green photoreceptors is clear.

Bees are, of course, highly adept at learning colors, mostly for purposes of flower identification. However, target detection based on chromatic cues is accomplished from short distances (Giurfa et al., 1996, 1997), where targets subtend large visual angles and image motion is reduced. Moreover, bees reduce flight speed if forced to rely on chromatic cues for target detection (Spaethe et al., 2001). Why then do bumblebees invest in relatively fast photoreceptors for color vision? The answer may be more to do with perceptual constancy than color vision per se (Skorupski and Chittka, 2010). In conditions of dappled foliage

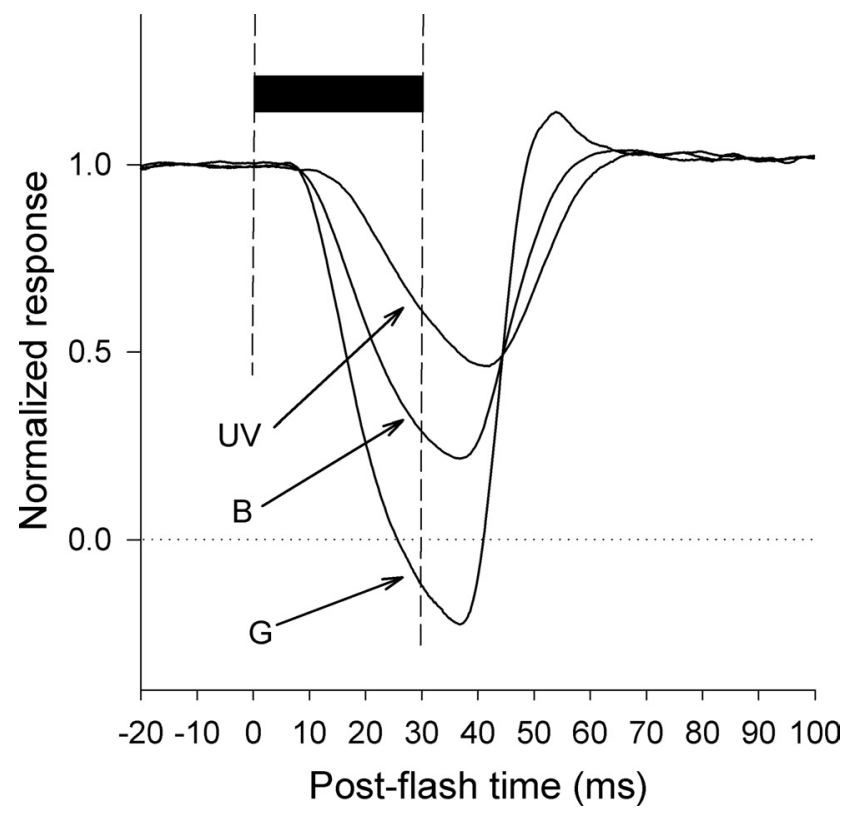

Figure 6. Averaged, normalized responses to $30 \mathrm{~ms}$ light-off pulses from green (G), blue (B), and UV photoreceptors. Each trace is pooled averaged data from five (green), four (blue), and three (UV) bees. Twenty to 30 light-off responses were averaged in each bee. Responses are normalized to the mean light-adapted membrane potential (i.e., 0 represents the dark resting potential; dotted line). The black bar represents the duration of the darkness pulse.

Table 3. Comparison of impulse response time to peak $\left(t_{\mathrm{p}}\right)$ and half-width $(\Delta t)$ for bumblebee photoreceptors with published photoreceptor data from other insect species

\begin{tabular}{lllll}
\hline Species & Spectral type & $t_{\mathrm{p}}(\mathrm{ms})$ & $\Delta t(\mathrm{~ms})$ & Reference \\
\hline Cricket & Unspecified & 22.8 & 16.6 & Howard et al., 1983 \\
Locust & Unspecified & 21.9 & 16.2 & Howard et al., 1983 \\
Mantid & Unspecified & 18.1 & 13.3 & Howard et al., 1983 \\
Hoverfly & Unspecified & 16.5 & 12.8 & Howard et al., 1983 \\
Bumblebee & UV & 15.7 & 12.3 & Present study \\
Dragonfly & Unspecified & 17.5 & 11.4 & Howard et al., 1983 \\
Bumblebee & Blue & 13.9 & 9.8 & Present study \\
Calliphora & R7-R8 & 12.4 & 8.8 & Anderson and Laughlin, 2000 \\
Bumblebee & Green & 12.5 & 7.9 & Present study \\
Housefly & Unspecified & 12 & 6.4 & Howard et al., 1983 \\
Calliphora & R1-R6 & 7.2 & 4.7 & Anderson and Laughlin, 2000 \\
Calliphora & R1-R6 & 7 & 4.3 & Tatler et al., 2000 \\
\hline
\end{tabular}

Measurements of $t_{\mathrm{p}}$ ranged from $9.7 \pm 0.2 \mathrm{~ms}$ at $19^{\circ} \mathrm{C}$ to $5.4 \pm 0.1 \mathrm{~ms}$ at $34^{\circ} \mathrm{C}$; tabulated values are estimated from response at $22^{\circ} \mathrm{C}$, illustrated in Fig $1 A$ of the reference.

${ }^{a}$ Female flies.

${ }^{b}$ Male flies.

(as may occur, for example, moving from flower to flower within an inflorescence), brightness varies more randomly than color (Mollon, 1989). During movement this will lead to luminance flicker, and this degrades the reliability of luminance far more than chromaticity signals (Maximov, 2000). Therefore, an opponent process that compares signals from spectrally different photoreceptors can filter out variability in luminance while yielding a chromaticity signal as a useful byproduct. However, such a mechanism implies the demand for frequency matching, which in turn would constrain the response times of chromatic photoreceptors to be kept within some reasonable limit of achromatic response times.

\section{Temporal and spatial resolution of color vision}

Honeybees and bumblebees use both chromatic and achromatic cues for target detection, but spatial acuity is reduced when only 
chromatic contrast is available (Giurfa et al., 1996; Spaethe et al., 2001). However, honeybees have finer color discrimination than bumblebees, but much poorer acuity for color detection; a target presenting only chromatic contrast must subtend a visual angle of at least $15^{\circ}$ for honeybee detection, whereas a bumblebee can detect a similar target of $\sim 3^{\circ}$ (Giurfa et al., 1996; Dyer et al., 2008; Wertlen et al., 2008). Our measurements of bumblebee photoreceptor noise (Fig. 3C) are considerably higher than honeybee (Vorobyev et al., 2001), which probably explains finer color discrimination in honeybees. The finer spatial acuity for chromatic contrast in bumblebees may partly be explained by their larger eyes and consequent superior optics (Spaethe and Chittka, 2003; Wertlen et al., 2008). However, our estimates of the noise time constant, $\tau$ (Eq. 3), are shorter than those for honeybee (Vorobyev et al., 2001), suggesting that bumblebees have faster eyes. This may also contribute to increased spatial resolution, since spatial resolution during movement depends on temporal frequency response (Land, 1999).

\section{Parallel visual processing}

Anatomical and physiological data from bees point to a picture of parallel visual processing reminiscent of that in primates. Signals from green photoreceptors are transmitted to the first optic ganglion, the lamina, whereas blue and UV photoreceptor axons pass directly to medulla, where photoreceptor signals can potentially be compared for chromatic computations (Menzel and Blakers, 1976; Yang et al., 2004; Morante and Desplan, 2008; Takemura et al., 2008; Paulk et al., 2009a). Chromatic processing is localized to specific layers of the lobula (the third-order visual neuropil) and has been observed in higher brain structures such as the protocerebrum (Paulk et al., 2008, 2009b). Spike latencies in central brain seem to be $\sim 10-30 \mathrm{~ms}$. For comparison, spiking latencies in cat visual cortex are of the order of 35-100 ms (Nowak and Bullier, 1997).

Changizi (2008) has made the intriguing suggestion that neuronal delays are not a handicap for complex nervous systems. The problem is not to map time-changing neural representations, with minimal delay, to a corresponding time series of real-world events. Rather, the nervous system needs a predictive representation of the world to guide behavior-whether this representation is itself phase locked to real time is irrelevant (Dennett and Kinsbourne, 1992). In this way, animals with large nervous systems (in terms of neural number) need not be in a hurry to represent the world, so long as the representations have sufficient predictive utility. If so, this could be relevant to the interesting problem of complex behavior generated by nervous systems based on an architecture that seems to be vastly more economical with neuronal number (Chittka and Niven, 2009). Animals such as the honeybee seem capable of impressive cognitive feats with brains composed of slightly fewer than one million neurons (Menzel and Giurfa, 2001). We are tempted to speculate that the question of neuronal number in CNS architecture may in fact be related to predictive strategies where processing delays are not the (most immediate) constraint. Bees, on the other hand, with a CNS architecture that is not based on modules of hundreds of thousands of neurons, do not have this excess processing capacity and are therefore forced to live closer to "real time." This would be in keeping with the fact that insect photoreceptors can be an order of magnitude faster than vertebrate cones. It would also explain why chromatic flicker fusion frequency in bees $(\sim 80 \mathrm{~Hz})$ (Srinivasan and Lehrer, 1985) approaches what might be expected based on photoreceptor response times, whereas in humans it is much lower, and certainly not limited by photoreceptor or even cortical neuronal response time (Williams et al., 2004). Perhaps this is why large areas of the human cortex can respond to chromatic flicker that is perceptually invisible and behaviorally undetectable (Jiang et al., 2007). Such a dissociation would seem less likely in a bee.

\section{References}

Anderson JC, Hardie RC (1996) Different photoreceptors within the same retina express unique combinations of potassium channels. J Comp Physiol A Neuroethol Sens Neural Behav Physiol 178:513-522.

Anderson JC, Laughlin SB (2000) Photoreceptor performance and the coordination of achromatic and chromatic inputs in the fly visual system. Vision Res 40:13-31.

Baylor DA (1987) Photoreceptor signals and vision. Proctor lecture. Invest Ophthalmol Vis Sci 28:34-49.

Baylor DA, Hodgkin AL (1974) Changes in time scale and sensitivity in turtle photoreceptors. J Physiol 242:729-758.

Baylor DA, Nunn BJ, Schnapf JL (1987) Spectral sensitivity of cones of the monkey Macaca fascicularis. J Physiol 390:145-160.

Boynton RM (1979) Human color vision. New York: Holt, Rinehart and Winston.

Briscoe AD, Chittka L (2001) The evolution of color vision in insects. Annu Rev Entomol 46:471-510.

Changizi MA (2008) The trade-off between speed and complexity. Behav Brain Sci 31:203.

Chittka L, Niven JE (2009) Are bigger brains better? Curr Biol 19:R995R1008.

Dennett D, Kinsbourne M (1992) Time and the observer: the where and when of consciousness in the brain. Behav Brain Sci 15:183-247.

Dodge FA Jr, Knight BW, Toyoda J (1968) Voltage noise in limulus visual cells. Science 160:88-90.

Dunn FA, Lankheet MJ, Rieke F (2007) Light adaptation in cone vision involves switching between receptor and post-receptor sites. Nature 449:603-606.

Dyer AG, Spaethe J, Prack S (2008) Comparative psychophysics of bumblebee and honeybee colour discrimination and object detection. J Comp Physiol A Neuroethol Sens Neural Behav Physiol 194:617-627.

Giurfa M, Lehrer M (2001) Honeybee vision and floral displays: from detection to close-up recognition. In: Cognitive ecology of pollination (Chittka L, Thomson JD, eds), pp 61-82. Cambridge: UP.

Giurfa M, Vorobyev M, Kevan P, Menzel R (1996) Detection of coloured stimuli by honeybees: minimum visual angles and receptor specific contrasts. J Comp Physiol A Neuroethol Sens Neural Behav Physiol 178:699-709.

Giurfa M, Vorobyev M, Brandt R, Posner B, Menzel R (1997) Discrimination of coloured stimuli by honeybees: alternative use of achromatic and chromatic signals. J Comp Physiol A Neuroethol Sens Neural Behav Physiol 180:235-243.

Howard J, Dubs A, Payne R (1984) The dynamics of phototransduction in insects: a comparative study. J Comp Physiol A Neuroethol Sens Neural Behav Physiol 154:707-718.

Howard J, Blakeslee B, Laughlin SB (1987) The intracellular pupil mechanism and photoreceptor signal: noise ratios in the fly Lucilia cuprina. Proc R Soc Lond B 231:415-435.

Jacobs GH (2003) Comparative psychology of vision. In: Handbook of psychology: biological psychology (Gallagher M, Nelson RJ, eds), pp 47-70: Hoboken, NJ: Wiley.

Jiang Y, Zhou K, He S (2007) Human visual cortex responds to invisible chromatic flicker. Nat Neurosci 10:657-662.

Kraft TW (1988) Photocurrents of cone photoreceptors of the goldenmantled ground squirrel. J Physiol 404:199-213.

Land MF (1999) Motion and vision: why animals move their eyes. J Comp Physiol A Neuroethol Sens Neural Behav Physiol 185:341-352.

Laughlin SB, Weckstrom M (1993) Fast and slow photoreceptors: a comparative study of the functional diversity of coding and conductances in the diptera. J Comp Physiol A Neuroethol Sens Neural Behav Physiol 172:593-609.

Laughlin SB, de Ruyter van Steveninck RR, Anderson JC (1998) The metabolic cost of neural information. Nat Neurosci 1:36-41.

Lehrer M, Campan R (2005) Generalization of convex shapes by bees: what are shapes made of? J Exp Biol 208:3233-3247.

Lehrer M, Srinivasan MV, Zhang SW, Horridge GA (1988) Motion cues 
provide the bee's visual world with a third dimension. Nature 332:356-357.

Livingstone MS, Hubel DH (1987) Psychophysical evidence for separate channels for the perception of form, color, movement and depth. J Neurosci 7:3416-3468.

Maximov VV (2000) Environmental factors which may have led to the appearance of colour vision. Philos Trans R Soc Lond B Biol Sci 355:1239-1242.

Menzel R, Blakers M (1976) Color receptors in the bee eye: morphology and spectral sensitivity. J Comp Physiol A Neuroethol Sens Neural Behav Physiol 108:11-33.

Menzel R, Giurfa M (2001) Cognitive architecture of a mini-brain: the honeybee. Trends Cogn Sci 5:62-71.

Mollon JD (1989) “Tho' she kneel'd in that place where they grew ...": the uses and origins of primate color vision. J Exp Biol 146:21-38.

Morante J, Desplan C (2008) The color-vision circuit in the medulla of Drosophila. Curr Biol 18:553-565.

Niven JE, Anderson JC, Laughlin SB (2007) Fly photoreceptors demonstrate energy-information trade-offs in neural coding. PLoS Biology 5:e116

Nowak LG, Bullier J (1997) The timing of information transfer in the visual system. In: Extrastriate visual cortex in primates (Rockland KS, Kaas JH, Peters A, eds), pp 205-241. New York: Plenum.

Paulk AC, Phillips-Portillo J, Dacks AM, Fellous JM, Gronenberg W (2008) The processing of color, motion, and stimulus timing are anatomically segregated in the bumblebee brain. J Neurosci 28:6319-6332.

Paulk AC, Dacks AM, Gronenberg W (2009a) Color processing in the medulla of the bumblebee (apidae: Bombus impatiens). J Comp Neurol 513:441-456.

Paulk AC, Dacks AM, Phillips-Portillo J, Fellous JM, Gronenberg W (2009b) Visual processing in the central bee brain. J Neurosci 29:9987-9999.

Regan BC, Julliot C, Simmen B, Viénot F, Charles-Dominique P, Mollon JD (2001) Fruits, foliage and the evolution of primate colour vision. Philos Trans R Soc Lond B Biol Sci 356:229-283.

Schneeweis DM, Schnapf JL (1999) The photovoltage of macaque cone photoreceptors: adaptation, noise, and kinetics. J Neurosci 19:1203-1216.

Skorupski P, Chittka L (2010) Is colour cognitive? Optics Laser Tech. Advance online publication. Retrieved February 25, 2010. doi: 10.1016/j.optlastec. 2008.12.015.
Skorupski P, Spaethe J, Chittka L (2006) Visual search and decision making in bees: time, speed and accuracy. Int J Comp Psychol 19:342-357.

Skorupski P, Döring TF, Chittka L (2007) Photoreceptor spectral sensitivities in island and mainland populations of the bumblebee, Bombus terrestris. J Comp Physiol A Neuroethol Sens Neural Behav Physiol 193:485-494.

Snowden R, Thompson P, Troscianko T (2006) Basic vision. Oxford: Oxford UP.

Spaethe J, Chittka L (2003) Interindividual variation of eye optics and single object resolution in bumblebees. J Exp Biol 206:3447-3453.

Spaethe J, Tautz J, Chittka L (2001) Visual constraints in foraging bumblebees: flower size and color affect search time and flight behavior. Proc Natl Acad Sci U S A 98:3898-3903.

Srinivasan M, Lehrer M (1985) Temporal resolution of colour vision in the honeybee. J Comp Physiol A Neuroethol Sens Neural Behav Physiol 157:579-586.

Srinivasan MV, Lehrer M (1988) Spatial acuity of honeybee vision and its spectral properties. J Comp Physiol A Neuroethol Sens Neural Behav Physiol 162:159-172.

Takemura SY, Lu Z, Meinertzhagen IA (2008) Synaptic circuits of the Drosophila optic lobe: the input terminals to the medulla. J Comp Neurol 509:493-513.

Vorobyev M, Brandt R, Peitsch D, Laughlin SB, Menzel R (2001) Colour thresholds and receptor noise: behaviour and physiology compared. Vision Res 41:639-653.

Wakakuwa M, Stavenga DG, Arikawa K (2007) Spectral organization of ommatidia in flower-visiting insects. Photochem Photobiol 83:27-34.

Wertlen AM, Niggebrügge C, Vorobyev M, Hempel de Ibarra N (2008) Detection of patches of coloured discs by bees. J Exp Biol 211:2101-2104.

Williams PE, Mechler F, Gordon J, Shapley R, Hawken MJ (2004) Entrainment to video displays in primary visual cortex of macaque and humans. J Neurosci 24:8278-8288.

Yang EC, Lin HC, Hung YS (2004) Patterns of chromatic information processing in the lobula of the honeybee, Apis mellifera L. J Insect Physiol 50:913-925.

Zeil J, Kelber A, Voss R (1996) Structure and function of learning flights in ground-nesting bees and wasps. J Exp Biol 199:245-252. 